\title{
Antibody EPR3864 is specific for ERG genomic fusions in prostate cancer: implications for pathological practice
}

\author{
Geert JLH van Leenders ${ }^{1}$, Joost L Boormans ${ }^{2}$, Cornelis J Vissers ${ }^{1}$, A Marije Hoogland ${ }^{1}$, \\ Anke AJWM Bressers ${ }^{1}$, Bungo Furusato ${ }^{3}$ and Jan Trapman ${ }^{1}$ \\ ${ }^{1}$ Department of Pathology, Josephine Nefkens Institute, Erasmus Medical Center, Rotterdam, \\ The Netherlands; ${ }^{2}$ Department of Urology, Erasmus Medical Center, Rotterdam, The Netherlands and \\ ${ }^{3}$ International Pathology Laboratory, Tokyo, Japan
}

\begin{abstract}
Genomic rearrangements involving genes encoding erythroblast transformation-specific transcription factors are commonly present in prostate cancer. The TMPRSS2-ERG gene fusion that leads to ERG overexpression occurs in $\sim 70 \%$ of prostate cancers. Implementation of fusion gene detection in pathological practice, however, has been hampered by the lack of reliable ERG antibodies. The objective of this study was first to compare ERG immunohistochemistry using the recently described antibody EPR3864 with ERG mRNA by quantitative PCR and, second, to investigate ERG immunohistochemistry in diagnostic prostate cancer needle biopsies. We analyzed 41 primary prostate adenocarcinomas obtained by radical prostatectomy and 83 consecutive prostate cancer needle biopsies. In the prostatectomy specimens, immunohistochemical ERG expression was highly concordant with the ERG mRNA overexpression (sensitivity $100 \%$ and specificity $85 \%$ ). ERG overexpression was due to TMPRSS2-ERG gene fusion in all cases. ERG protein expression was identified in $51 / 83$ adenocarcinomas $(61 \%)$ on needle biopsies. ERG expression was more frequent in tumors infiltrating $\geq 2$ needle biopsies $(P<0.001)$ or occupying $\geq 50 \%$ of a single biopsy $(P=0.018)$. Expression of ERG also occurred in 11/21 (52\%) high-grade prostate intraepithelial neoplasia lesions. In 5/87 (6\%) needle biopsies containing benign secretory glands, weak ERG staining was focally observed. In all of these cases, respective glands were adjacent to adenocarcinomas. In conclusion, immunohistochemistry for ERG strongly correlated with ERG mRNA overexpression and was specific for prostate cancer on needle biopsies. Therefore, ERG immunohistochemistry is an important adjunctive tool for pathophysiological studies on ERG gene fusions, and might support the pathological diagnosis of adenocarcinoma in a subset of prostate needle biopsies. Modern Pathology (2011) 24, 1128-1138; doi:10.1038/modpathol.2011.65; published online 15 April 2011
\end{abstract}

Keywords: ERG; fusion; immunohistochemistry; prostate cancer; translocation

Genomic rearrangements of androgen-regulated, prostate-specific genes to genes encoding erythroblast transformation-specific (ETS) transcription factors characterize the majority of human prostate cancers. Fusion of transmembrane protease, serine 2 (TMPRSS2) to v-ets erythroblastosis virus E26 oncogene homolog $(E R G)$ occurs in $40-70 \%$, representing the most common genetic alteration in prostate cancer. ${ }^{1-3}$ Less frequently, solute carrier family 45, member 3 (SLC45A3) and N-myc down-

Correspondence: Dr GJLH van Leenders, MD, PhD, Department of Pathology, Josephine Nefkens Institute, Erasmus Medical Center, PO Box 2040, 3000 CA Rotterdam, The Netherlands.

E-mail: g.vanleenders@erasmusmc.nl

Received 16 November 2010; revised 3 February 2011; accepted 3 February 2011; published online 15 April 2011 stream regulated 1 (NDRG1) have been reported as ERG fusion partners. ${ }^{4-6}$ Moreover, fusions of ETS variant (ETV) family members ETV1, ETV4 and ETV5 to over 10 different partner genes are present in $\sim 10 \%$ of tumors. ${ }^{7-10}$ In $20-50 \%$ of tumors, no ETS rearrangements can be detected, with a proportion of fusion gene-negative prostate cancers showing overexpression of serine peptidase inhibitor, Kazal type 1 (SPINK1). ${ }^{11}$

Since its discovery, several groups have studied the relation of TMPRSS2-ERG, as determined by quantitative PCR (QPCR) or fluorescence in situ hybridization (FISH), with pathological disease parameters, clinical behavior and histological features with varying outcome. ${ }^{12-19}$ Because of its high prevalence, detection of $E R G$ can be of utility in the diagnosis of prostate cancer in daily pathological 
practice. Application of FISH for this purpose is, however, time consuming and suffers from difficulty in interpretation, especially if only a few atypical glands are present in the tissue sample. Reliable QPCR for routine detection of ERG overexpression needs frozen tissue sections containing a high percentage of prostate cancer. Lack of a reliable ERG antibody has significantly hampered studies on the pathophysiological function of $E R G$ rearrangements in prostate carcinogenesis and on their putative role in tumor progression.

Recently, Park et $a l^{20}$ compared ERG protein expression by immunohistochemistry, using antibody EPR3864, with ERG rearrangement by FISH in prostate cancer paraffin-embedded tissues. In the current study, we correlated ERG immunohistochemistry with ERG mRNA overexpression caused by gene fusion in primary prostate cancer obtained by radical prostatectomy. Moreover, we applied ERG immunohistochemistry in diagnostic needle biopsies to investigate its utility in pathological practice. The characterization of an antibody specific for $E R G$ genomic rearrangements will highly facilitate studies on gene fusion in prostate carcinogenesis and tumor progression, and might support the pathological diagnosis of adenocarcinoma in a subset of prostate needle biopsies.

\section{Materials and methods}

\section{Clinical Samples}

For molecular analysis of ERG expression, we included 41 patients treated by radical prostatectomy for prostate cancer in Erasmus Medical Center between 1985 and 2001. None of the patients had undergone radiation or hormonal therapy before surgery. Immediately after arrival at the Pathology department, small samples from the putative tumor area were dissected and freshly frozen in liquid nitrogen. All frozen tissues were stained by hematoxylin/eosin and the percentage of malignant cells was semiquantitatively estimated; in this study, only specimens containing at least $70 \%$ tumor cells were included. After formalin fixation, prostatectomy specimens were entirely embedded for diagnostic pathological evaluation, at which the Gleason score, pT stage (WHO 2004) and surgical margin status were routinely determined. We correlated genomic ERG expression as assessed by QPCR on the fresh-frozen sample, with ERG immunohistochemical expression of the paraffin slide containing the largest tumor volume of the same patient. Owing to the retrospective nature of this study, we were unable to determine the topographic relation between the area of which the frozen sample was derived and the paraffin block containing the largest tumor volume.

For immunohistochemical studies on needle biopsies $(N=95)$, we selected all consecutive patients diagnosed with prostate cancer in Erasmus Medical Center $(N=77)$ and Haven Hospital $(N=18)$ in Rotterdam, between January and September 2010. Needle biopsies were prompted by elevated prostate-specific antigen (PSA) level, abnormal digital rectal examination or transrectal ultrasound. Needle biopsies from Erasmus Medical Center were embedded in a separate container each, allowing optimal determination of the number and the percentage of positive biopsies. At Haven Hospital, multiple biopsies were collected in two containers representing both prostatic halves. The number of positive biopsies, percentage of tumor infiltration in each biopsy, modified Gleason score and presence of high-grade prostate intraepithelial neoplasia were routinely recorded by a urological pathologist $(\mathrm{GvL})$. The first two features were only determined for needle biopsies obtained in Erasmus Medical Center. Use of samples for research purposes was approved by the Erasmus Medical Center Medical Ethics Committee according to the Medical Research Involving Human Subjects Act (MEC-2004-261).

\section{QPCR}

RNA from prostatectomy samples was isolated using RNA-Bee (Campro Scientific, Berlin, Germany). Total RNA was reverse transcribed using M-MLV reverse transcriptase (Invitrogen, Carlsbad, CA, USA) and an oligo dT12 primer. QPCR reactions were performed in Power SYBR Green PCR Master Mix $(25 \mu \mathrm{l})$, containing $0.33 \mu \mathrm{M}$ forward and reverse primer in an ABI Prism 7700 Sequence Detection System (Applied Biosystems, Carlsbad, CA, USA). Amplified products were quantified relative to hydroxymethylbilane synthase (HMBS; formerly $P B G D$ ). Sequences of the primers used are summarized in Table 1. To exclude artifacts caused by the

Table 1 Primers used for QPCR analysis

\begin{tabular}{lll}
\hline Target & Forward $5^{\prime}-3^{\prime}$ & Reverse $5^{\prime}-3^{\prime}$ \\
\hline HMBS & CATGTCTGGTAACGGCAATG & GTACGAGGCTTTCAATGTTG \\
TRG & TGCTCAACCATCTCCTTCCA & TGGGTTTGTCTTCCGCTCT \\
TMPRSS2 (exon 0$)-E R G$ & GAGCTAAGCAGGAGGCGGA & CATCAGGAGAGTTCCTGAG \\
TMPRSS2 (exon 1$)-E R G$ & GACTACTTCTACTCCACCAG & CATCAGGAGAGTTCCTTGAG \\
ETV1 & GAGCTAAGCAGGAGGCGGA & CATCAGGAGAGTTCCTTGAG \\
& CATACCAACGGCGAGGATCA & TGGAGAAAAGGGCTTCTGGA \\
\hline
\end{tabular}


use of one housekeeping gene, we also carried out QPCR for glyceraldehyde-3-phosphate dehydrogenase $(G A P D H)$ as a reference (Hs99999905_m1; Applied Biosystems). QPCR for $E R G$ was considered positive if $E R G / H M B S$ or $E R G / G A P D H$ ratio was higher than 5.0.

\section{Immunohistochemistry}

Formalin-fixed, paraffin-embedded tissue sections $(5 \mu \mathrm{m})$ from radical prostatectomy specimens and needle biopsies were cut and mounted on aminoacetylsilane-coated slides (Starfrost, Berlin, Germany). Sections were deparaffinized in xylene and dehydrated in ethanol. Endogenous peroxidase activity was quenched by incubation with $1 \%$ hydrogen peroxide in methanol for $20 \mathrm{~min}$. For all antibodies, microwave $(700 \mathrm{~W})$ pretreatment in tris(hydroxymethyl)aminomethane-EDTA (pH 9.0) was performed for $15 \mathrm{~min}$. Slides were incubated with rabbit monoclonal ERG antibody (clone EPR3864; 1:100; Epitomics, Burlingame, CA, USA) overnight at $4{ }^{\circ} \mathrm{C}$, followed by chromogenic visualization using the EnVision system (DAKO, Glostrup, Denmark). A subset of five needle biopsies was stained for FLI1 (1:1500; clone C19; Santa Cruz Biotechnology, Santa Cruz, CA, USA) for $30 \mathrm{~min}$ at room temperature. Negative control experiments were done by omitting the first antibody. Tissues were counterstained with hematoxylin. Finally, the slides were dehydrated, cleared in xylene and mounted in Malinol (Chroma-Gesellschaft, Köngen, Germany).

The intensity of ERG immunohistochemical expression was scored as negative (0; no staining), weak $(1+$; only visible at high magnification), moderate $(2+$; visible at low magnification) and strong $(3+$; striking at low magnification). Nuclear reactivity of the antibody in endothelial cells was used as internal control for the staining procedure. ${ }^{20}$ In case of staining heterogeneity, the highest level was used for statistical analysis.

\section{Statistical Analysis}

Associations between expression of ERG mRNA and ERG protein expression in surgical specimens were evaluated by the Pearson's $\chi^{2}$-test or the MannWhitney $U$-test. Immunohistochemical expression of ERG in needle biopsies was correlated with age, PSA, Gleason score, number of positive biopsies and maximum percentage of tumor infiltration, using Students' $t$-test and Pearson's $\chi^{2}$-test. Statistical analyses were performed using the Statistical Package for Social Sciences, version 17.0 (SPSS, Chicago, IL, USA), with a significance level of 0.05 (twotailed probability).

\section{Results}

\section{Correlation of ERG Immunohistochemistry with ERG Gene Fusions}

To validate the properties of the ERG antibody, we first compared immunohistochemical staining with ERG mRNA expression in archival primary prostate cancers for which both tumor RNA and paraffin tissue was available $(N=41)$. The mean age of the prostatectomy patients was 61.9 years (range 49.172.8 years). The Gleason score was 6 in 20 (49\%), 7 in $13(32 \%)$ and $\geq 8$ in $8(19 \%)$ cases, respectively. Fourteen (34\%) tumors were organ confined (pT2), and $27(66 \%)$ cases showed extra-prostatic expansion (pT3/4). Surgical margins were positive in 19 $(46 \%)$ cases. The clinicopathological characteristics of the prostate cancers at prostatectomy and their immunohistochemical ERG expression are summarized in Table 2. ERG mRNA overexpression was detected in 28 (68\%) samples. Of the $13(32 \%)$

Table 2 Clinicopathological characterization of prostate cancer in radical prostatectomy specimens $(N=41)$ in association with ERG immunohistochemistry

\begin{tabular}{|c|c|c|c|c|}
\hline Parameter & Number (\%) & ERG positive & ERG negative & $\mathrm{P}$-value \\
\hline Age (years), mean (range) & $61.9(49.1-2.8)$ & $61.1(49.1-9.4)$ & $64.1(49.6-72.8)$ & 0.2 \\
\hline \multicolumn{5}{|l|}{ Gleason score } \\
\hline 6 & $20(49)$ & 16 & 4 & \\
\hline $7(3+4)$ & $9(22)$ & 7 & 2 & \\
\hline $7(4+3)$ & $4(10)$ & 3 & 1 & 0.4 \\
\hline$\geq 8$ & $8(19)$ & 4 & 4 & \\
\hline \multicolumn{5}{|l|}{ pT stage } \\
\hline 2 & $14(34)$ & 9 & 5 & \\
\hline $3 a$ & $15(36)$ & 13 & 2 & \\
\hline $3 \mathrm{~b}$ & $6(15)$ & 3 & 3 & 0.3 \\
\hline 4 & $6(15)$ & 5 & 1 & \\
\hline \multicolumn{5}{|l|}{ Surgical margin } \\
\hline Negative & $22(54)$ & 16 & 6 & \\
\hline Positive & $19(46)$ & 14 & 5 & 0.9 \\
\hline
\end{tabular}


tumors without ERG mRNA overexpression, 4 samples $(10 \%)$ demonstrated overexpression of ETV1 transcripts. As depicted in Table 3, immunohistochemical staining of ERG was highly concordant with the presence of $E R G$ overexpression, as assayed by QPCR (sensitivity 100\%; specificity $85 \%$; and Pearson's $\left.\chi^{2}, P<0.001\right)$. Only two tumors

Table 3 Correlation of ERG expression determined by QPCR on RNA from frozen prostate cancer obtained by radical prostatectomy and corresponding immunohistochemistry of paraffin-embedded tissue

\begin{tabular}{lrc}
\hline ERG & \multicolumn{2}{c}{ QPCR } \\
\cline { 2 - 3 } & Negative & Positive \\
\hline $\begin{array}{l}\text { Immunohistochemistry } \\
\text { Negative } \\
\text { Positive }\end{array}$ & 11 & 0 \\
\hline
\end{tabular}

stained positive for ERG without high $E R G$ mRNA expression. In all samples showing ERG mRNA overexpression, it was caused by fusion of $E R G$ to TMPRSS2 (data not shown). High immunohistochemical ERG expression was not only found in tumors that expressed the major TMPRSS2-ERG fusion transcript, TMPRSS2(exon 1)-ERG, but also in a sample that showed exclusively high expression of the less frequent TMPRSS2(exon 0)-ERG transcript (Figure 1). ${ }^{19}$ To exclude artifacts induced by the housekeeping gene $H M B S$, we also carried out QPCR for GAPDH as a reference, which resulted in an identical selection of $E R G$-positive cases. The median value of ERG/GAPDH in ERG-positive cases was 28.3 (range 10.5-59.2) as compared with $1.3(0.3-4.7)$ in negative tumors.

As shown in Table 4, prostate cancer with strong immunohistochemical ERG expression $(3+)$ had a significantly higher relative ERG transcript level than tumors with combined weak $(1+)$ and moder-

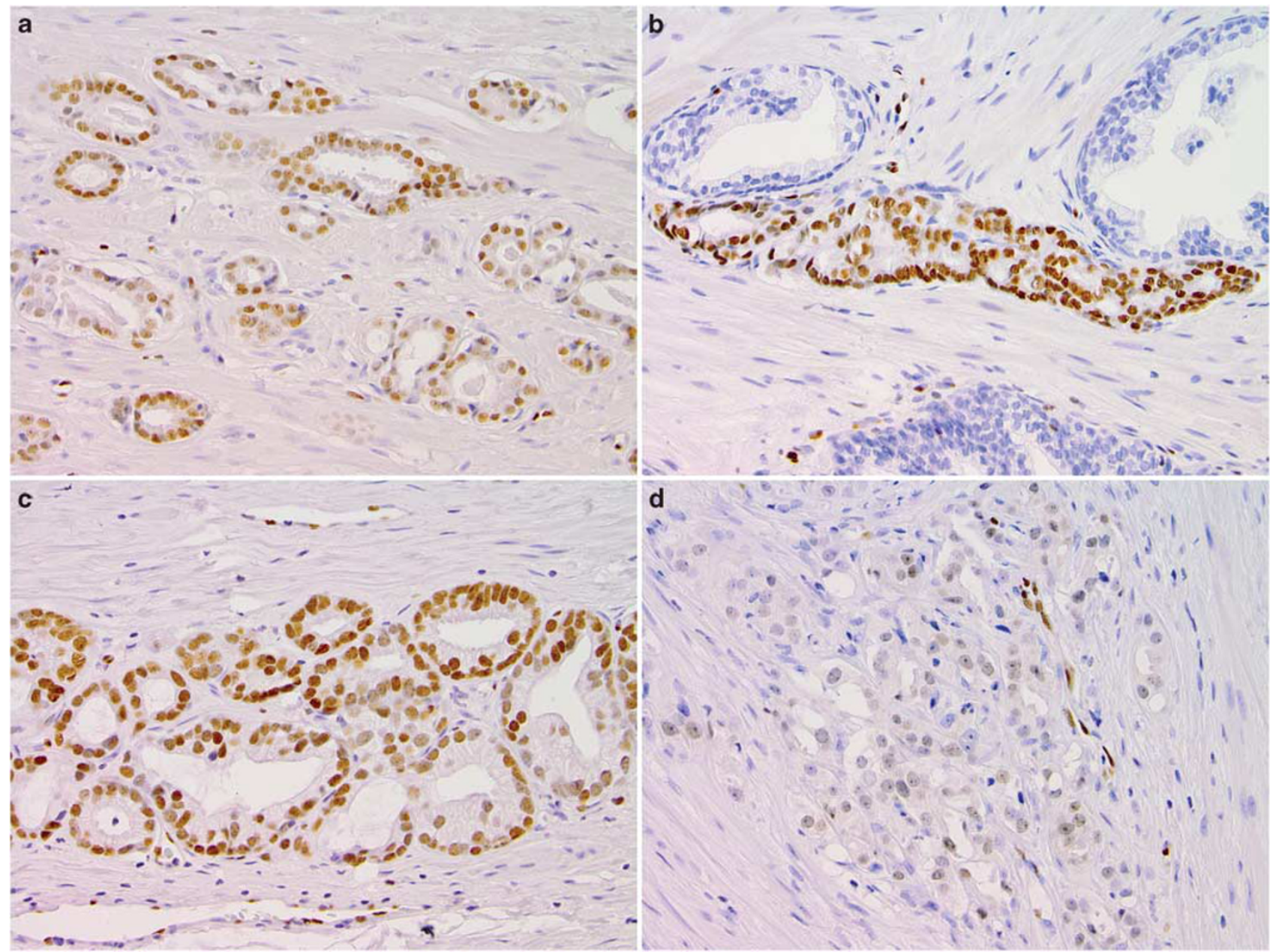

Figure 1 ERG protein expression in prostate cancer at radical prostatectomies as assessed by immunohistochemistry was highly concordant with overexpression of $E R G$ mRNA as assayed by quantitative PCR. ERG staining was present in prostate cancer containing TMPRSS2(exon 1)-ERG fusion transcripts (a), TMPRSS2(exon 0)-ERG fusion transcripts (b) and tumors with both transcripts (c). ERG protein expression was significantly higher in cancer with high relative ERG mRNA levels than in tumors with low mRNA levels. Weak $(1+)$ immunohistochemical ERG staining in prostate cancer with low ERG overexpression $(E R G /$ hydroxymethylbilane synthase $(H M B S)=12 ; \mathbf{d})$ as compared with strong $(3+)$ immunohistochemical ERG staining in a tumor with high $E R G$ levels $(E R G / H M B S=79.7$; a); both tumors had TMPRSS2(exon 1)-ERG fusion transcripts. ERG; original magnifications a-d $\times 200$. 
Table 4 Correlation of ERG protein expression with relative $E R G$ mRNA levels in prostate cancer $(N=39)$

\begin{tabular}{lrc}
\hline Immunohistochemistry & $\mathrm{N}$ & $\begin{array}{c}\text { QPCR ERG/HMBS, } \\
\text { median (range) }\end{array}$ \\
\hline 0 & 11 & $1.4(0.2-3.6)$ \\
1 & 11 & $25.0(8.3-45.7)$ \\
2 & 10 & $24.0(11.1-53.6)$ \\
3 & 7 & $43.3(9.8-79.7)$
\end{tabular}

Strong (3+) ERG protein expression correlated with higher expression of $E R G$ as compared with combined weak (1+) and moderate $(2+)$ immunohistochemistry $(P<0.04)$. Both cases with false-positive ERG protein expression were excluded from analysis.

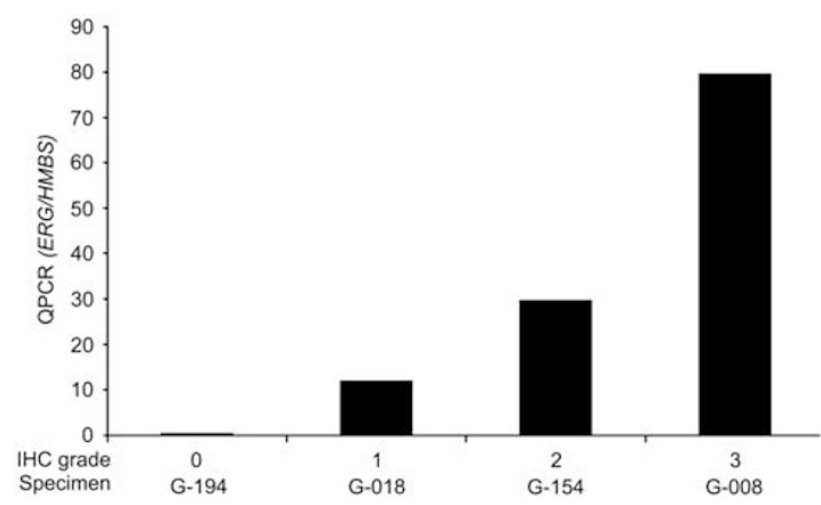

Figure 2 Association of semiquantitative ERG immunohistochemical expression (IHC grade) with quantitative PCR $E R G /$ hydroxymethylbilane synthase ratio in four representative prostate cancers at radical prostatectomy.

ate $(2+)$ staining (Mann-Whitney, $P<0.04)$. There was no significant difference of $E R G$ mRNA levels in tumors with weak $(1+)$ and moderate $(2+)$ immunohistochemical ERG expression $(P=0.75)$. For four representative cases, the association of ERG immunohistochemistry and ERG/HMBS ratio is depicted in Figure 2. Expression levels of ERG mRNA in a separate group of benign prostate tissues derived from prostatectomy specimens (median value $E R G / H M B S 1.3 ; N=17$ ) were similar to that in $E R G$-negative prostate cancer. In this relatively small series, there was no correlation of ERG immunohistochemistry (Table 2) or ERG overexpression (data not shown), with clinicopathological parameters at radical prostatectomy.

\section{ERG Expression in Prostate Cancer Needle Biopsies}

To investigate whether immunohistochemical ERG expression can aid in diagnosing adenocarcinoma on needle biopsies, we studied its expression in 95 consecutive malignant biopsies collected in 2010. In 12 cases, no residual cancer was detected because of renewed sectioning of the paraffin block. The clinical context that prompted the diagnostic needle biopsies in the remaining patients $(N=83)$ was: participation in the European Randomized Screening study for Prostate Cancer $(N=28)$, clinical suspicion of prostate cancer $(N=39)$ and follow-up in the scope of active surveillance $(N=16) .^{21}$ The patients' mean age was 68.2 years (range 39.7-79.2 years). The median PSA level was $6.7 \mathrm{ng} / \mathrm{ml}$ (range $0.8-745 \mathrm{ng} / \mathrm{ml}$ ). Gleason score on needle biopsy was 6 in $48(58 \%), 7$ in $22(27 \%)$ and $\geq 8$ in $13(15 \%)$ tumors. ERG protein expression was identified in 51 adenocarcinomas (61\%). Expression of ERG was strong $(3+)$ in $37(45 \%)$, moderate in 8 $(10 \%)$, weak in $6(7 \%)$ and negative in $32(38 \%)$ tumors (Figure 3). Immunohistochemical ERG expression was not related to age, PSA level or Gleason score at biopsy, as shown in Table 5. For analysis of ERG expression in relation to tumor burden, we excluded all patients containing multiple biopsy specimens in one container (see Materials and methods). ERG expression was identified more frequently if $\geq 2$ biopsies were infiltrated with adenocarcinoma (Pearson's $\chi^{2}, P<0.001$ ) and if $\geq 50 \%$ of the biopsy specimen was affected $(P=0.018)$. This indicates that the sensitivity of ERG protein expression is highest in cases with more abundant tumor infiltration. In fact, when we analyzed ERG expression in the subgroup of patients with one positive needle biopsy infiltrated with $\leq 25 \%$ of tumor, which represents the most relevant cases for adjuvant immunohistochemical staining $(N=22)$, seven tumors $(32 \%)$ were positive.

In the current analysis, we scored ERG immunohistochemistry 'positive' if expression occurred in the whole tumor or in any area of the tumor. Expression heterogeneity was, however, observed within the same needle biopsy or between multiple biopsies included in one container. In 2 of $70(3 \%)$ prostate cancer foci, positive and negative ERG immunohistochemistry occurred within the same needle biopsy, whereas in $2 / 13$ cases $(15 \%)$, heterogeneity occurred in different needle biopsies included within the same container.

Nuclear antibody reactivity in endothelial cells served as internal control for an adequate immunohistochemical staining procedure. As the ERG antibody is reported to show cross-reactivity with FLI1, we studied FLI1 expression in a set of needle biopsies with ERG-expressing adenocarcinoma. In these cases, weak FLI1 expression was observed in endothelial cells, but prostate adenocarcinoma was negative. Although staining by the ERG antibody of endothelial cells might therefore be, partially, due to cross-reactivity with FLI1, its reactivity in these cells demonstrates the effectiveness of immunohistochemical technique (Figure 4).

\section{High-Grade Prostate Intraepithelial Neoplasia}

In the series analyzed, a total of 21 needle biopsies contained high-grade prostate intraepithelial 

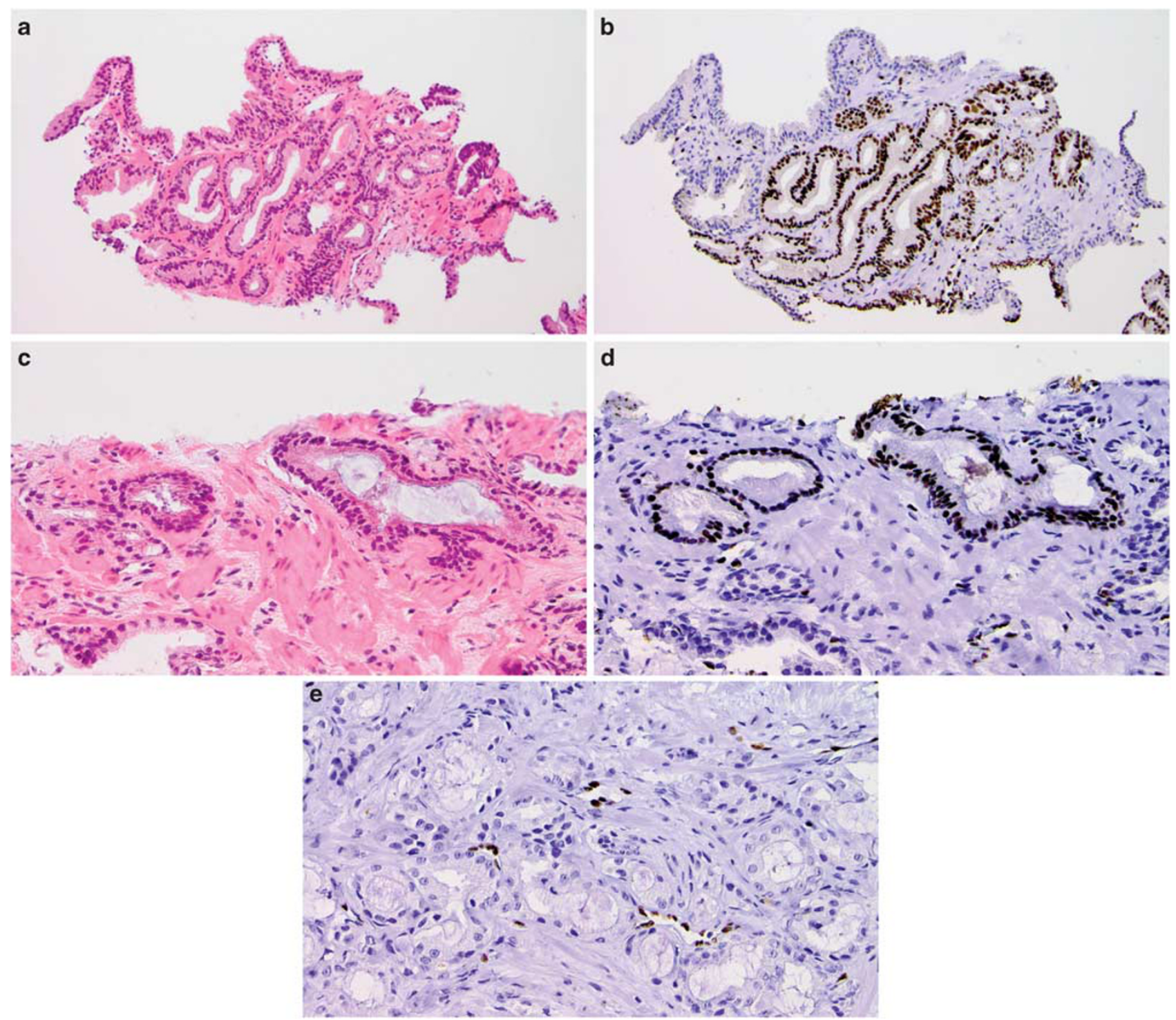

Figure 3 Strong nuclear immunohistochemical ERG expression was present in small foci of prostate cancer (a-d) on needle biopsies. Note that ERG expression is completely absent in adjacent benign secretory glands. In one-third of prostate cancers, no ERG expression was detected (e), where endothelial cells served as an internal control. (a, c) Hematoxylin/eosin; (b, d, e) ERG; original magnifications a, b $\times 100, \mathbf{c}-\mathbf{e} \times 200$.

neoplasia. Expression of ERG was detected in 11 cases $(52 \%)$. In all these cases, ERG expression occurred in luminal epithelial cells within the same gland. ERG expression in prostate intraepithelial neoplasia was highly concordant with its expression in adjacent adenocarcinoma (Pearson's $\chi^{2}, P<0.001$ ). Of the 19 cases in which prostate intraepithelial neoplasia and adenocarcinoma were present in the same slide, both were positive $(N=10)$ or negative $(N=8)$ for ERG; in one case, adenocarcinoma was positive and adjacent prostate intraepithelial neoplasia negative. In two cases, prostate intraepithelial neoplasia was present without residual adenocarcinoma, of which one was positive and one negative for ERG expression (Figure 5a and b).

\section{Benign Prostate Glands}

A total of 87 needle biopsies contained benign secretory glands, which were negative for ERG expression in 82 cases (94\%). In five biopsies $(6 \%)$, focal and weak $(1+)$ ERG expression was detected in histologically benign glands (Figure 5c). Immunohistochemical ERG expression was not detected in any of the 40 needle biopsies with atrophic glands. The morphologically normal glands that demonstrated weak ERG expression were all located adjacent to or in between malignant glands. We did not encounter any case with ERG expression in benign glands spatially distant from malignant glands. 
Table 5 Relation of ERG immunohistochemistry with clinicopathological parameters in prostate cancer needle biopsies $(N=83)$

\begin{tabular}{|c|c|c|c|}
\hline & ERG negative & ERG positive & Significance (P-value) \\
\hline Overall expression & $32(39 \%)$ & $51(61 \%)$ & NA \\
\hline Age (years; mean) & 68.5 & 68.1 & 0.81 \\
\hline PSA (ng/ml; median) & 6.2 & 6.8 & 0.57 \\
\hline \multicolumn{4}{|l|}{ Gleason score } \\
\hline 6 & $21(25 \%)$ & $27(33 \%)$ & \\
\hline 7 & $5(6 \%)$ & $17(21 \%)$ & $6 v s \geq 7$ \\
\hline $8-10$ & $6(7 \%)$ & $7(8 \%)$ & 0.09 \\
\hline \multicolumn{4}{|c|}{ Number of positive biopsies* } \\
\hline 1 & $19(28 \%)$ & $8(11 \%)$ & \\
\hline 2 & $1(1 \%)$ & $16(23 \%)$ & 1 vs $\geq 2$ \\
\hline$>2$ & $8(11 \%)$ & $18(26 \%)$ & $<0.001$ \\
\hline \multicolumn{4}{|l|}{ Percentage of tumor* } \\
\hline$\leq 5$ & $7(10 \%)$ & $7(10 \%)$ & \\
\hline $5-25$ & $9(13 \%)$ & $13(18 \%)$ & $<50 \%$ vs $\geq 50 \%$ \\
\hline $25-50$ & $10(14 \%)$ & $5(7 \%)$ & 0.018 \\
\hline $50-75$ & $1(2 \%)$ & $10(14 \%)$ & \\
\hline $75-100$ & $1(2 \%)$ & $7(10 \%)$ & \\
\hline
\end{tabular}

For ERG expression analyses with regard to number of positive needle biopsies and maximum tumor percentage per needle biopsy $\left({ }^{*}\right)$, only cases from Erasmus Medical Center $(N=70)$ were included. If multiple categories were present, the most significant association was depicted.
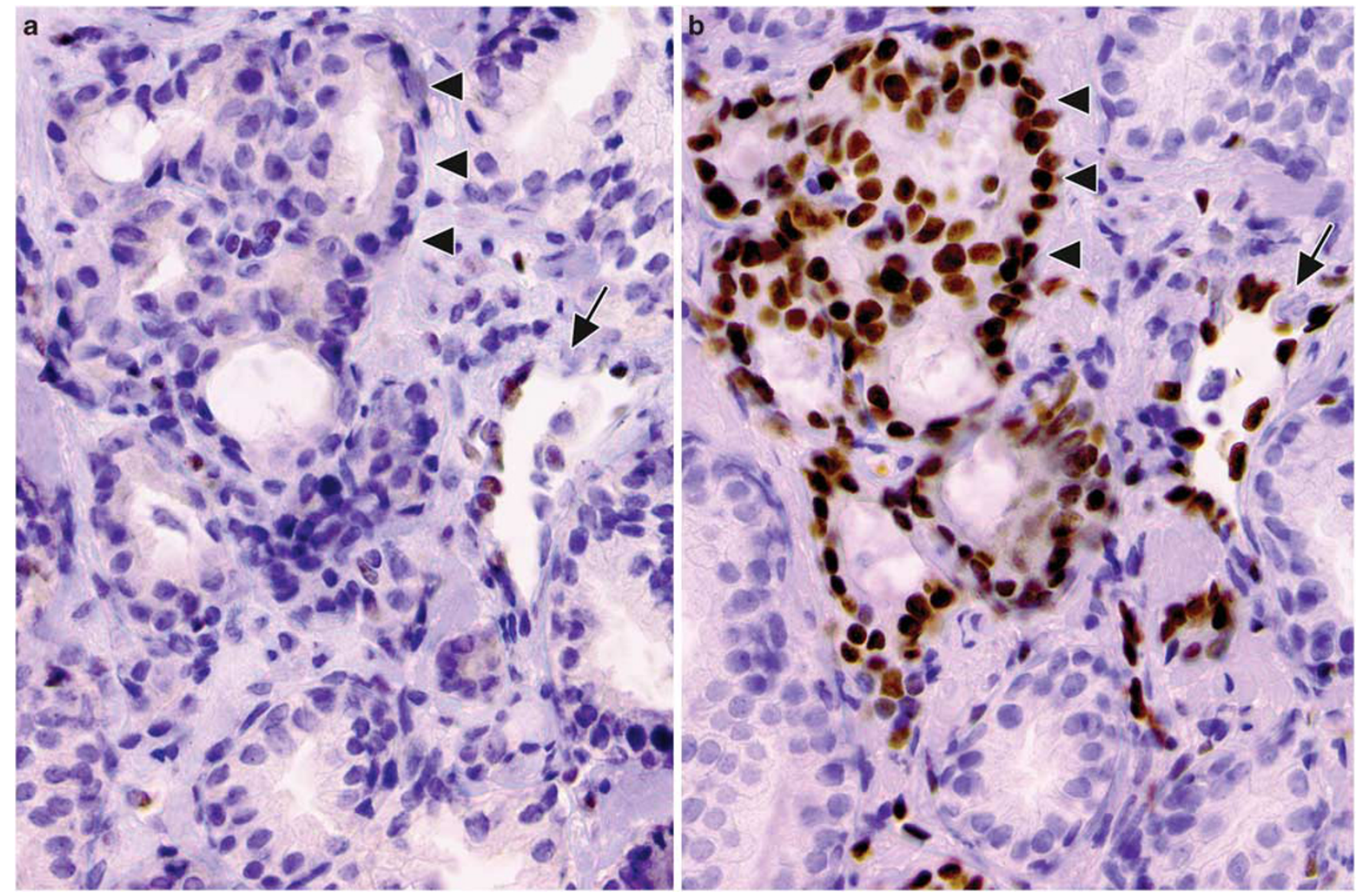

Figure 4 Weak nuclear expression of FLI1 was present in endothelial cells, whereas both benign prostate epithelial and adenocarcinoma were negative (a). Both adenocarcinoma and endothelial cells showed strong ERG expression (b). Arrows indicate endothelial cells; arrowheads indicate prostate cancer. (a) FLI1; (b) ERG; original magnification $\times 200$. 


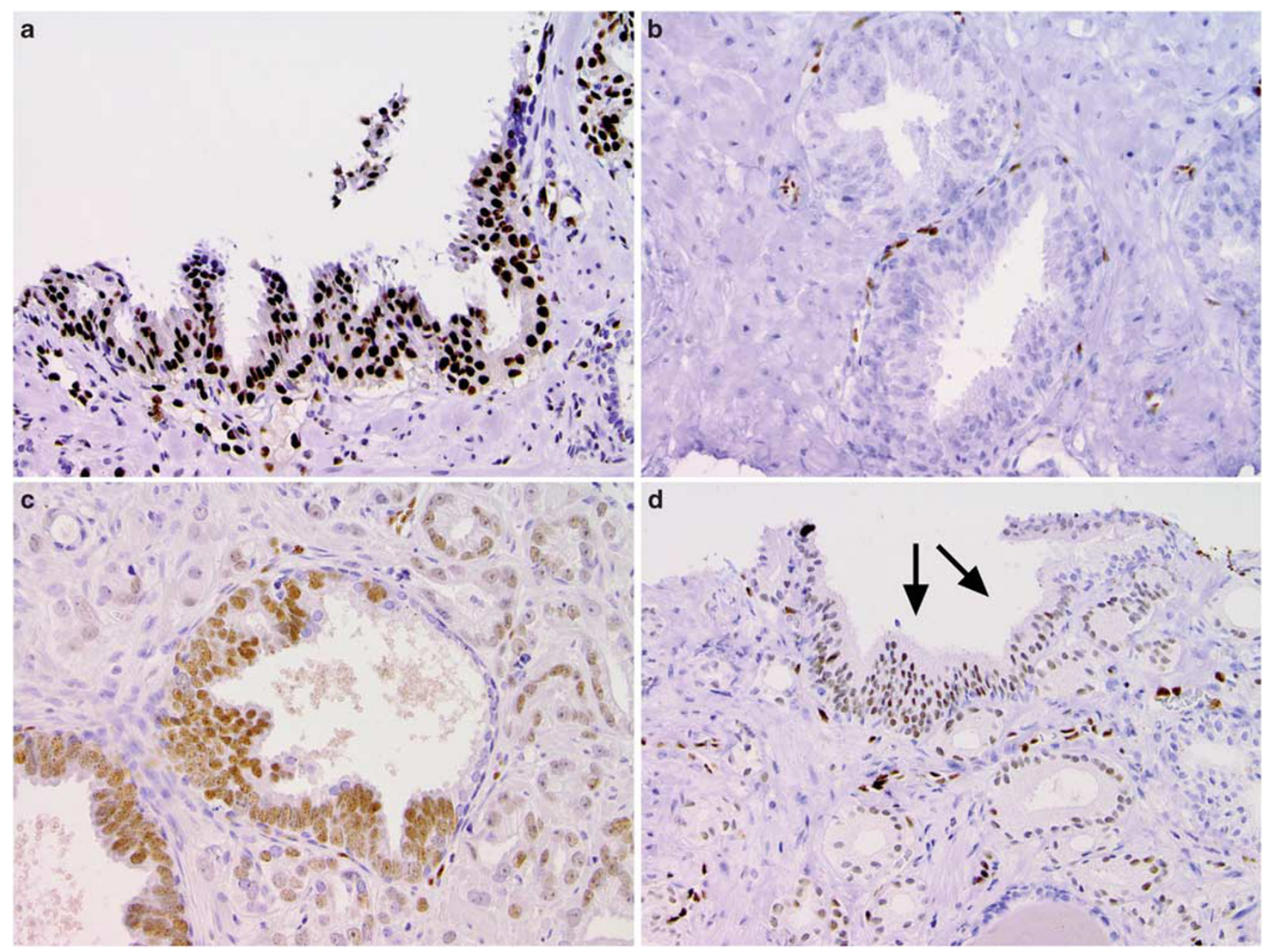

Figure 5 ERG was expressed in a subset of high-grade prostate intraepithelial neoplasia (a) on needle biopsies, where other lesions were negative (b). Immunohistochemical ERG expression clearly demarcated prostate intraepithelial neoplasia expanding in a benign secretory gland (c; radical prostatectomy). Although ERG immunohistochemistry is negative in the vast majority of non-malignant prostate glands, ERG staining was sporadically detected in secretory glands (arrows) that did not fulfill all histological criteria for high-grade prostate intraepithelial neoplasia (d). (a-d) ERG; original magnification $\times 200$.

If needle biopsies containing adenocarcinoma and benign prostate glands were both included, the sensitivity of ERG expression was $61 \%$, specificity $94 \%$, positive predictive value $92 \%$ and negative predictive value $72 \%$.

\section{Discussion}

Overexpression of $E R G$ fusion transcripts have been detected in $\sim 70 \%$ of primary prostate cancer. $^{1,12,15,17,19,22-27}$ Therefore, it is an important functional tumor marker. TMPRSS2-ERG fusion has been associated with some histological features i.c. (in casu) blue-tinged mucin, cribriform growth, intraductal spread, macronucleoli and signet-ring cells, particularly if more than one of these features were present. ${ }^{18} E R G$ gene rearrangements seem to occur less frequently in pure ductal or mixed acinarductal prostate cancer, as compared with conventional acinar tumors. ${ }^{28}$ Recently, a rabbit anti-ERG monoclonal antibody was described showing excellent concordance of positive ERG immunohistochemistry with presence of $E R G$ fusion, as detected by FISH, irrespective of the $E R G$ fusion partner. ${ }^{20}$

In concordance with these recent findings, we demonstrated in the present study that the ERG protein expression strongly correlated with ERG mRNA overexpression in primary prostate cancer. Moreover, we found that the tumors with highest ERG mRNA overexpression showed strongest ERG immunohistochemistry. In only two cases, ERG protein expression was detected in the absence of ERG mRNA overexpression. Because RNA was isolated from a different tumor section than the tissue slides used for immunohistochemistry, this apparent discrepancy could be explained by intra/ intertumoral heterogeneity of genomic rearrangements, as has been reported in about a quarter of prostate cancer cases. ${ }^{29,30}$ Alternatively, false-positive expression could be due to cross-reactivity of the EPR3864 antibody with the ERG-related ETS 
protein FLI1, as has been communicated by the manufacturer, although FLI1 mRNA expression was low in all samples analyzed (data not shown). The high sensitivity of ERG protein expression for prostate cancer, as independently found in both studies, indicates that ERG immunohistochemistry could be an adjunctive tool for diagnosing prostate cancer on needle biopsies.

Although the histological diagnosis of prostate cancer is usually straightforward, immunohistochemistry can be applied in the evaluation of difficult cases. Visualization of basal cells using antibodies targeting cytoplasmic basal, high-molecular-weight keratins or nuclear p63 is the traditional and most helpful tool supporting or rejecting prostate cancer diagnosis. Moreover, enhanced cytoplasmic expression of alpha-methylacyl CoAracemase (AMACR) is more common in prostate cancer than in benign glands. Nevertheless, various problems might occur in the interpretation of immunohistochemistry in atypical glandular foci on needle biopsies. ${ }^{31,32}$ For instance, partial atrophy on needle biopsy has an immunophenotype identical to adenocarcinoma in $24 \%$ of cases. $^{31,33}$ Atypical adenomatous hyperplasia/adenosis, benign prostate tissue and crowding of secretory glands demonstrate AMACR expression in 17, 21 and 64\% of cases, respectively. ${ }^{31,34,35}$ On the other hand, conventional prostate cancer is negative for AMACR in $\sim 20 \%$, whereas its pseudohyperplastic and foamy gland variants do not reveal expression in $30-40 \% .^{36,37}$ Ultimately, aberrant expression of high-molecular-weight keratins in prostate cancer has been reported in rare cases. ${ }^{38-40}$ Therefore, there is an ongoing need for the discovery and clinical implementation of novel biomarkers that can support or reject prostate cancer diagnosis in difficult cases.

In this study, we investigated ERG protein expression in prostate cancer on needle biopsies, which clinically represent the most relevant tissue specimens for implementation of novel biomarkers. Overall expression of ERG occurred in $61 \%$ of adenocarcinomas with $94 \%$ specificity, indicating that its expression strongly favors a diagnosis of malignancy in difficult cases. The majority of highgrade prostate intraepithelial neoplasias adjacent to ERG-positive adenocarcinoma were also ERG positive. ERG protein expression was only focally and weakly expressed in histologically benign glands. These benign glands were all adjacent to malignant glands.

Immunohistochemical ERG expression was more prevalent in prostate intraepithelial neoplasia than expected, based on previous rearrangement studies by FISH that showed ERG overexpression ranging from $0-17 \% .^{1,13,29,30,41}$ This discordance might be caused by underestimation of ERG rearrangements, as the specific pathological features of prostate intraepithelial neoplasia can be appreciated less well by FISH. The high frequency of ERG expression in high-grade intraepithelial neoplasia does not support the hypothesis that ERG overexpression is involved in the transition of prostate intraepithelial neoplasia to adenocarcinoma. ${ }^{42}$ In fact, it would support a hypothesis that ERG is instrumental in the formation of high-grade intraepithelial neoplasia from normal prostate epithelium. This is also suggested by the weak ERG expression in about $6 \%$ of benign secretory glands that did not fulfill all histopathological criteria for high-grade prostate intraepithelial neoplasia and were adjacent to adenocarcinoma. In concordance with the sporadic occurrence of $E R G$ rearrangements in histologically benign glands, our results support the theory that early molecular aberrations precede pathologically recognizable preneoplasia. ${ }^{43}$

Some important points for clinical practice have to be taken into account in interpreting our results. First, ERG was evaluated in needle biopsies containing both adenocarcinomas and benign glands, and not in an independent set of actual benign prostate biopsies. This might have caused a bias in the analysis of ERG specificity in non-malignant tissues. Nevertheless, the absence of ERG expression in benign glands distant from adenocarcinoma within the same biopsy and in multiple benign biopsies concomitantly present within the same container implicates that benign glands indeed only sporadically express ERG protein. Second, the present study indicated that ERG staining is less frequent in single small foci of adenocarcinoma, being positive in one-third of cases with tumor infiltration in $\leq 25 \%$ of a single needle biopsy. Therefore, it remains the question to what extend ERG immunohistochemistry is complementary to already available antibodies in diagnostic classification of small atypical glandular lesions. The lower percentage of small tumors staining for ERG as compared with more voluminous cancers might partially be explained by the methodology used in the current study. If ERG expression was detected in a heterogeneously staining prostate cancer, this case was labeled as positive. Interestingly, one could also speculate that absence of ERG protein expression is a marker for indolent disease, based on its less frequent presence in small tumors, as has also been suggested by others. ${ }^{12,14,44,45}$ The observation that such relation was not reflected by an association between ERG expression and pathological parameters at prostatectomy might be explained by a selection bias of relatively more aggressive tumors in the latter group. As only frozen specimens containing at least $70 \%$ tumor tissue were selected, minute, possibly indolent, tumors were not included in the prostatectomy series. However, other explanations cannot be excluded.

In conclusion, ERG protein expression as detected by immunohistochemistry with the ERG3864 antibody is highly concordant with $E R G$ mRNA expression in primary prostate cancer. Moreover, nuclear ERG expression was identified in $61 \%$ of 
adenocarcinomas on diagnostic needle biopsies. ERG expression is also present in about half of the high-grade prostate intraepithelial neoplasia lesions, but it is only rarely found in isolated benign secretory glands. Therefore, ERG immunohistochemistry highly facilitates future studies on the role of ERG overexpression due to gene fusion in clinical prostate cancer.

\section{Disclosure/conflict of interest}

The authors declare no conflict of interest.

\section{References}

1 Tomlins SA, Rhodes DR, Perner S, et al. Recurrent fusion of TMPRSS2 and ETS transcription factor genes in prostate cancer. Science 2005;310:644-648.

2 Clark JP, Cooper CS. ETS gene fusions in prostate cancer. Nat Rev Urol 2009;6:429-439.

3 Kumar-Sinha C, Tomlins SA, Chinnaiyan AM. Recurrent gene fusions in prostate cancer. Nat Rev Cancer 2008;8:497-511.

4 Pflueger D, Rickman DS, Sboner A, et al. N-myc downstream regulated gene 1 (NDRG1) is fused to ERG in prostate cancer. Neoplasia 2009;11:804-811.

5 Han B, Mehra R, Dhanasekaran SM, et al. A fluorescence in situ hybridization screen for E26 transformation-specific aberrations: identification of DDX5-ETV4 fusion protein in prostate cancer. Cancer Res 2008;68:7629-7637.

6 Esgueva R, Perner S, LaFargue CJ, et al. Prevalence of TMPRSS2-ERG and SLC45A3-ERG gene fusions in a large prostatectomy cohort. Mod Pathol 2010;23: 539-546.

7 Helgeson BE, Tomlins SA, Shah N, et al. Characterization of TMPRSS2:ETV5 and SLC45A3:ETV5 gene fusions in prostate cancer. Cancer Res 2008;68:73-80.

8 Tomlins SA, Mehra R, Rhodes DR, et al. TMPRSS2:ETV4 gene fusions define a third molecular subtype of prostate cancer. Cancer Res 2006;66: 3396-3400.

9 Hermans KG, Bressers AA, van der Korput HA, et al. Two unique novel prostate-specific and androgenregulated fusion partners of ETV4 in prostate cancer. Cancer Res 2008;68:3094-3098.

10 Hermans KG, van der Korput HA, van Marion R, et al. Truncated ETV1, fused to novel tissue-specific genes, and full-length ETV1 in prostate cancer. Cancer Res 2008;68:7541-7549.

11 Tomlins SA, Rhodes DR, Yu J, et al. The role of SPINK1 in ETS rearrangement-negative prostate cancers. Cancer Cell 2008;13:519-528.

12 Demichelis F, Fall K, Perner S, et al. TMPRSS2:ERG gene fusion associated with lethal prostate cancer in a watchful waiting cohort. Oncogene 2007;26: 4596-4599.

13 Perner S, Mosquera JM, Demichelis F, et al. TMPRSS2ERG fusion prostate cancer: an early molecular event associated with invasion. Am J Surg Pathol 2007;31: 882-888.

14 Nam RK, Sugar L, Yang W, et al. Expression of the TMPRSS2:ERG fusion gene predicts cancer recurrence after surgery for localised prostate cancer. Br J Cancer 2007;97:1690-1695.

15 Nam RK, Sugar L, Wang Z, et al. Expression of TMPRSS2:ERG gene fusion in prostate cancer cells is an important prognostic factor for cancer progression. Cancer Biol Ther 2007;6:40-45.

16 Wang J, Cai Y, Ren C, et al. Expression of variant TMPRSS2/ERG fusion messenger RNAs is associated with aggressive prostate cancer. Cancer Res 2006; 66:8347-8351.

17 Gopalan A, Leversha MA, Satagopan JM, et al. TMPRSS2-ERG gene fusion is not associated with outcome in patients treated by prostatectomy. Cancer Res 2009;69:1400-1406.

18 Mosquera JM, Perner S, Demichelis F, et al. Morphological features of TMPRSS2-ERG gene fusion prostate cancer. J Pathol 2007;212:91-101.

19 Hermans KG, Boormans JL, Gasi D, et al. Overexpression of prostate-specific TMPRSS2(exon 0)-ERG fusion transcripts corresponds with favorable prognosis of prostate cancer. Clin Cancer Res 2009;15:6398-6403.

20 Park K, Tomlins SA, Mudaliar KM, et al. Antibodybased detection of ERG rearrangement-positive prostate cancer. Neoplasia 2010;12:590-598.

21 Schroder FH, Hugosson J, Roobol MJ, et al. Screening and prostate-cancer mortality in a randomized European study. N Engl J Med 2009;360:1320-1328.

22 Albadine R, Latour M, Toubaji A, et al. TMPRSS2-ERG gene fusion status in minute (minimal) prostatic adenocarcinoma. Mod Pathol 2009;22:1415-1422.

23 Fine SW, Gopalan A, Leversha MA, et al. TMPRSS2$E R G$ gene fusion is associated with low Gleason scores and not with high-grade morphological features. Mod Pathol 2010;23:1325-1333.

24 Mehra R, Tomlins SA, Shen R, et al. Comprehensive assessment of TMPRSS2 and ETS family gene aberrations in clinically localized prostate cancer. Mod Pathol 2007;20:538-544.

25 Mosquera JM, Mehra R, Regan MM, et al. Prevalence of TMPRSS2-ERG fusion prostate cancer among men undergoing prostate biopsy in the United States. Clin Cancer Res 2009;15:4706-4711.

26 Rajput AB, Miller MA, De Luca A, et al. Frequency of the TMPRSS2:ERG gene fusion is increased in moderate to poorly differentiated prostate cancers. J Clin Pathol 2007;60:1238-1243.

27 Saramaki OR, Harjula AE, Martikainen PM, et al. TMPRSS2:ERG fusion identifies a subgroup of prostate cancers with a favorable prognosis. Clin Cancer Res 2008;14:3395-3400.

28 Lotan TL, Toubaji A, Albadine R, et al. TMPRSS2-ERG gene fusions are infrequent in prostatic ductal adenocarcinomas. Mod Pathol 2009;22:359-365.

29 Furusato B, Gao CL, Ravindranath L, et al. Mapping of TMPRSS2-ERG fusions in the context of multi-focal prostate cancer. Mod Pathol 2008;21:67-75.

30 Zhang S, Pavlovitz B, Tull J, et al. Detection of TMPRSS2 gene deletions and translocations in carcinoma, intraepithelial neoplasia, and normal epithelium of the prostate by direct fluorescence in situ hybridization. Diagn Mol Pathol 2010;19: 151-156.

31 Herawi M, Parwani AV, Irie J, et al. Small glandular proliferations on needle biopsies: most common benign mimickers of prostatic adenocarcinoma sent in for expert second opinion. Am J Surg Pathol 2005;29:874-880. 
32 Wolters $\mathrm{T}$, van der Kwast TH, Vissers CJ, et al. False-negative prostate needle biopsies: frequency, histopathologic features, and follow-up. Am J Surg Pathol 2010;34:35-43.

33 Wang W, Sun X, Epstein JI. Partial atrophy on prostate needle biopsy cores: a morphologic and immunohistochemical study. Am J Surg Pathol 2008;32:851-857.

34 Beach R, Gown AM, De Peralta-Venturina MN, et al. P504S immunohistochemical detection in 405 prostatic specimens including 376 18-gauge needle biopsies. Am J Surg Pathol 2002;26:1588-1596.

35 Yang XJ, Wu CL, Woda BA, et al. Expression of alpha-Methylacyl-CoA racemase (P504S) in atypical adenomatous hyperplasia of the prostate. Am J Surg Pathol 2002;26:921-925.

36 Magi-Galluzzi C, Luo J, Isaacs WB, et al. Alpha-methylacyl-CoA racemase: a variably sensitive immunohistochemical marker for the diagnosis of small prostate cancer foci on needle biopsy. Am J Surg Pathol 2003;27:1128-1133.

37 Zhou M, Jiang Z, Epstein JI. Expression and diagnostic utility of alpha-methylacyl-CoA-racemase (P504S) in foamy gland and pseudohyperplastic prostate cancer. Am J Surg Pathol 2003;27:772-778.

38 Ali TZ, Epstein JI. False positive labeling of prostate cancer with high molecular weight cytokeratin: p63 a more specific immunomarker for basal cells. Am J Surg Pathol 2008;32:1890-1895.
39 Osunkoya AO, Hansel DE, Sun X, et al. Aberrant diffuse expression of p63 in adenocarcinoma of the prostate on needle biopsy and radical prostatectomy: report of 21 cases. Am J Surg Pathol 2008;32: 461-467.

40 van Leenders GJ, Aalders TW, Hulsbergen-van de Kaa CA, et al. Expression of basal cell keratins in human prostate cancer metastases and cell lines. J Pathol 2001;195:563-570.

41 Mosquera JM, Perner S, Genega EM, et al. Characterization of TMPRSS2-ERG fusion high-grade prostatic intraepithelial neoplasia and potential clinical implications. Clin Cancer Res 2008;14:3380-3385.

42 Tomlins SA, Mehra R, Rhodes DR, et al. Integrative molecular concept modeling of prostate cancer progression. Nat Genet 2007;39:41-51.

43 Clark J, Attard G, Jhavar S, et al. Complex patterns of ETS gene alteration arise during cancer development in the human prostate. Oncogene 2008;27: 1993-2003.

44 Perner S, Demichelis F, Beroukhim R, et al. TMPRSS2:ERG fusion-associated deletions provide insight into the heterogeneity of prostate cancer. Cancer Res 2006;66:8337-8341.

45 Attard G, Clark J, Ambroisine L, et al. Duplication of the fusion of TMPRSS2 to ERG sequences identifies fatal human prostate cancer. Oncogene 2008;27: 253-263. 